\title{
Game-induced learning Effect: A Cognitive Neuroscience perspective
}

\author{
Rose Ru-Whui Lee ${ }^{1,2}$ Yao-Ting Sung ${ }^{3}$ Kuo-en Chang ${ }^{4}$ \\ ${ }^{1}$ Ph.D. student, Graduate Institute of Information and Computer Education, National \\ Normal Taiwan University, Taiwan \\ 2 Assistant Programmer, MEG Lab, Acdemia Sinica, Taiwan \\ ${ }^{3}$ Professor, Department of Educational Psychology and Counseling, National Taiwan \\ Normal University, Taiwan \\ ${ }^{4}$ Professor, Graduate Institute of Information and Computer Education, National Taiwan \\ Normal University, Taiwan
}

\begin{abstract}
There is a common belief among people that the video games for training and education used in our daily life could shape our brain. However, this belief has not been substantiated by solid scientific evidence. In particular, precisely how this shaping effect is to be measured has never been specified. This investigation looks into the game-induced learning effects by reviewing task-specific effects and their generalization in cognitive functions that relate to brain neural mechanism. Relevant literatures of the effects of training on the executive function of cognitive processes are carefully reviewed from the perspective of cognitive neuroscience research. Suggestions for the future directions of how to leverage technology for designing better games in order to enhance the efficiency of training/learning in education are proposed.
\end{abstract}

Keywords: game-induced learning effect, task-specific, cognitive neuroscience

\section{Introduction}

The digital environment associated with dynamic multi-sensory changes the way we learn and how we communicate with one another, and these changes shall give us a different perception of the world. Recent research in neuroscience has shown evidence that daily exposure in high technological environment gradually changes new neural pathways and rewires our brain, altering the state of our mind and the patterns of our behavior [1]. It is obvious that today's young people in teen-ages and 20s, the so called 'Digital Natives', who have been born in a world with computers, internet and cellphone, search information via online search engine intuitively and with sophistication, contrasting the way we 'digital immigrants' used to search information by material category bulletin in the real-world library. The modern technology devices, often constructed with a human-based engineering consideration, are designed more "smart" to fit the requirements of our daily life, and these biologically oriented applications with multisensory displays may have implications for making our brain-mind-behavior more naturally "smart." A phenomenon of the socalled Flynn Effect shows that the average scores of IQ test today's youngsters is higher than that of the early 20th century at an average rising rate of three points per decade [2]. One of the theoretical accounts of the Flynn effect focuses on the changes of our multi-media environment which stimulates and rewires our brain 
circuits and enhances our performance in a standard test. For example, today's youth immersing in the environment of action video games with the application of $2 \mathrm{D} / 3 \mathrm{D}$ presentations would contribute a great deal to enhance their visualspacial cognitive functions, as reflected in the positive correlation with higher average scores in CPM and SPM of Raven IQ Test (fluid intelligence) $[3,4]$.

If one takes the implications of the Flynn effect seriously, then it is important that a more powerful brain mechanism for cognitive functions in working memory, attention, and decision-making has to be developed and maintained. In this sense, higher speed and efficiency of information processing would be essential for our better daily life. In recent decades, numerous digital training programs had been developed as instructional tools to meet this need for enhancing the specific cognitive skills as well as remediating intervention of disability. Usually, the majority of these training programs were designed as video games due to their advantages of higher motivation and attention engagement of participants. This paper discusses the efficiency of gaming by two criteria: task-specific effect and their generalization in cognitive functions, and then make suggestions for how to design better digital games from the perspective of research in the cognitive neuroscience.

\section{Efficiency of Gaming}

\subsection{Training-induced Learning: Work- ing Memory}

Working memory is the ability to retain information for short period of time in order to comprehend and organize the incoming information. The amount of information that an individual can retain while processing information is called the working memory capacity. Traditionally, the working memory capacity has been thought to be constant. Recent experimental evidence from neuroscience studies has shown that the working memory capacity can be improved by training, consistent with the arguments based on the view of brain plasticity. These evidences came from studies of human subjects in both normal and disorders. Klingberg and his colleagues at the Karolinska Institu [5] used fMRI to investigate the changes in brain activities, which they proposed as induced by working memory training. They observed that after training, the increasing brain activities in the middle frontal gyrus and superior and inferior parietal cortices which had been reported to be related to working memory. They used the evidence to support their view of training-induced plasticity in the neural systems that underlie working memory in healthy adult human. There were also similar evidences shown in patients study to support the same conclusion. Haut et al.(2010)[6] compared the performance of schizophrenia patient controls, healthy controls, and patients treated as cognitive training; they found better performance after cognitive training was associated with increased activation of PFC (Prefrontal Cortex) regions, which was established as the regions (including dorsolateral prefrontal cortex, anterior cingulate and fronto-polar cortex) sub-serving attention and working memory. Some studies had observed similar training-induced effects in ADHD (Attention-deficit hyperactivity disorder) children (Klingberg et al. 2005)[7] and in stoke patients (Westerberg et al., 2007)[8] Moreover, Klingburg (2010)[9] also emphasizes the working memory capacity can be improved by adaptive and extended training which is associated with changes in brain activities in PFC regions; he also demonstrated transfer effects to non-trained working memory tasks, giving strong support to the notion of training-induced plasticity. In sum, he con- 
cludes that working memory training can be used as a remediating intervention for whom low working memory is a limiting factor for his or her academic performance or in everyday life.

\subsection{Task-Specific Effect}

Recent experiments which examined the influence of playing video games on cognitive abilities showed many task-specific effects of cognitive function resulting from playing video games, including the effects on attention, memory and executive control [10]. There are numerous examples of such effects in different aspects of cognitive function, such as visual skills in perceptual learning (visual modality, multi-sensory processing [11], earliest sensory of visual processing [12], contrast sensitivity) [13][14]), cognitive control (selective attention of task switching[15]), perceptual decision processing (suppression of distracting irrelevant information (focus attention) [16]), attention control (top-down attention control [17]. changing direction in strategy using [18], faster response time for visual search [19]), and production of motor responses (attention control within oculomoter system [20]).

\subsection{Generalization in cognitive function (transfer effect)}

The neural changes underlying the effects of training and learning are fundamental and critical to the changes at the behavioral level. Therefore, the positive effect resulting from successful intervention can be generalized to other related behaviors. For instance, Kjuala et al. (2001) demonstrated that reading difficulties can be ameliorated by special training programs even without linguistic materials and the training effect was demonstrated by faster reaction times to detecting sound changes. They also found that changes in brain ac- tivity at the auditory cortex were accompanied by improvement in reading skills [21]. In other words, the study highlighted the effect of training program in the activation of the underling neural mechanism which supports the higher lever cognitive tasks in general. The successful generalization as shown in this study has important implications for our aging society.

It is well known that we are facing a society problem due to the increase of aging population. It is also clear that the decline of cognitive control function in old adults is a serious threat to future society. The related issues have been widely discussed in both cross-sectional and longitudinal studies. The topics of how to train aging brain in order to counter the cognitive disorders are essential. Researchers such as Nouchjir et al. (2012) carefully pointed out that the beneficial effects of brain training game on cognition in elder people are selective; their study showed that the observed transfer to other cognitive functions occurred only in tasks including executive functions and processing speed but not on any global cognition and attention. Therefore, they suggested braining training games for elderly is useful for improving executive functions and processing speed in short term training[22]. Another investigation conducted by Muijden et al. (2012) evaluated transfer effect by Cognitive Test Battery (CTB) for working memory updating, set shifting, and response inhibition before and after the intervention of online cognitive training game. They found that the game group, as comparing to the non-game group in their study of healthy older adult age 60-73, had shown transfer effects on parts of the cognitive control functions including inhibition attention (stop-signal task), inductive reasoning (Raven-SPM), and selective attention (UFoV-3), which were not targeted by the intervention [23]. Such evidence 
has shown that the generalization of training effect can only be partially observed, and there is no "one fits for all" solution for enhancing cognitive control ability in aging society.

As we mentioned above, video games have been thought as a powerful tool for cognitive enhancement. Greeen and Bavelier (2012) reviewed the studies of the past decades and concluded that training on action video games produced learning that transfered well beyond the training task. They pointed out that the advantage on new task might not be observed immediately after game playing. They proposed that the true effect of action video game playing might be to enhance the ability to learn new task, especially in the application of rehabilitation paradigms [24]. Furthermore, Bavelier, Green, Pouget and Schrater (2012) optimistically encourage people to adopt complex training environments such as action video game to foster brain plasticity. They also emphasize learning on the application aspects of education, working training and rehabilitation through the life span [25]. However, There are levels of transfer that can be difficult to identify and quantify among transfer within the same domain, transfer to other cognitive constructs, and transfer to everyday life [26]. In contrast, Owen et al. (2010) conducted a six-week online study with 11,430 participants on broad range of cognitive functions in video game training among adults of ages from 18 to 60 . They observed task specific improvement but no transfer effect to untrained tasks, even though the tasks were cognitively related [27]. Besides, Melby-Lervag et al. (2012) indicated there was no convincing evidence of the generalization of working memory training to other skills, such as nonverbal and verbal ability, inhibitory processes in attention, word decoding, and arithmetic, in their meta-analytic review. They concluded that working memory training programs appeared to produce short-term, specific training effects that did not generalize[28]. There still seem to have a great deal of debate on this generalization issue. Further studies from different perspectives such as neurological, cognitive and behavioral assessment should carefully be carried out to clear up the ambiguity.

Eventually, evaluating generalization effect from the perspective of cognitive neuroscience, we need to more carefully examine the evidences on the changes of neural connections underlying the mechanism related to training and learning task which was developed for enhancing target behaviors.

\section{Conclusion and Suggestion}

The general game-induced learning effects are expected to be observed across the brain-mind-behavior scale consistently in the whole lifespan. Although, previous studies have found neruophysiological differences between gamers and nongamers, nevertheless, these are mostly limited to the issue of expert/novice comparisons. What we would like to do is to use neuroimaging to examine the effects by mapping the underlying brain mechanism of performance differences induced specifically by gaming. Boot and his colleagues (2011) discussed some methodological shortcomings for those studies which claim the positive gaming effects and then suggested the guidelines for more definitive tests of the effect of gaming on cognition, including covert recruitment strategy, adequate control of Placebo effects, differentiation from strategy changes, adequate baseline for transfer effects, and measurable improvement expectation from both experiment and control group, which is critical to recruiting, testing and reporting[29]. This cast the light on the possible specification to implement more studies with 
solid evidences in game-induced learning effect.

There is no doubt, in line with the trend of 'Mind-Brain-Education' evolution since 1990's, numerous brain plasticitybased training program were developed and commercialized to be adopted in family and schools. There is always need to carefully evaluate and criticize some Nurosmyths [26], such as right/left brain training, learning style preference (Visual, Auditory, or Kinesthetic_VAK), 'Brain Gym'_Brain button', 'Cross-crawl' (using pseudo-scientific terms to promote the idea that neural mechanisms can be influenced by specific physical exercises.) and so on. Goswami and her colleagues indicated the importance of cognitive neuroscience to be adopted to design and delivery of educational instruction to the wellbeing of our society [30][31][32]. In the future, leverage ICT engineering to design embedded formative assessment and neurofeedback interaction in the training/ learning program would be definitely play an important role in design and development. Bridging the cognitive neuroscience research to practice in education, clinic and ICT engineering is undoubted the main course in digit era.

\section{References}

[1] G. Small and G. Vgorgan. “ iBrain: Surviving the Technologyical Alteration of the Modern Mind." William Morrow, 2008.

[2] http://en.wikipedia.org/wiki/Intellige nce quotient.

[3] L. S. Colzato, N. C., van Wouwe, T. Lavender, and B. Hommel, "Intelligence and Cognitive Flexibity: Fluid Intelligence Correlates with Feature "UnBinding" Across Perception and Action." Psychon. Bull. Rev., 13, 1043-1048, 2006.

[4] L. S. Colzato, P. J. A. van Leeuwen, W. P. M. van den Wildenberg and B.
Hommel. "MOOD'd to Switch: Superi or Cognitive Flexibility in Players of First Person Shooter Games." Frontiers in Psychology, 1,8, 2010.

[5] P. J. Olesen, H. Westerberg, and T. Kingberg, "Increased Prefrontal and Parietal Activity after Training of Working Memory," nature neuroscience, 7(1), pp. 75-79, 2004.

[6] K. M. Haut, K. O. Lim, and Angus III Macdonald, "Prefrontal Cortical Changes following Cognitive Training in Patients with Chronic Schizophrenia: Effects of Practice, Generalization, and Specificity, "Neuropsychopharmacology, 35, pp. 1850$1859,2010$.

[7] T. Klingberg, E. Fernell, P. Olesen, M. Johnson, P. Gustafsson, K. Dahlström, C. G. Gillberg, H. Forssberg, and H. Westerberg, "Computerized training of Working Memory in Children with ADHD - a Randomized, Controlled Trial." Journal of the American Academy of Child and Adolescent Psychiatry, 44(2), pp. 177-186, 2005.

[8] H. Westerberg, H. Jacobaeus, T. Hirvikoski, P. Clevberger, M. L. Ostensson, A. Bartfai, and T. Klingberg, "Computerized Working Memory Training after Stroke-A Pilot Study." Brain Injury, 21(1),pp.21-29, 2007.

[9] T Kingberg, "Training and Plasticity of Working Memory," Trends in cognitive science, 14, pp. 317-324, 2010.

[10] W. R. Boot, A. F. Kramer, D. J. Si mons, and M. Fabiani, and G. Gratton, "The Effects of Video Game Playing on Attention, Memory, and Executive control." Acta Psychol (Amst), 129(3), pp.387-98, 2008.

[11] S. E. Donobue, M. G. Woldorff, and S. R. Mitroff, "Video Game Players Show Precise Multisensory Temporal Processing Abilities," Atten Percept Psychophs, 72(4), pp. 1120-1129, 2010. 
[12] G. L. West, S. A. Stevens, C. Pun, and J. Pratt, "Visuospatial Experience Modulates Attentional Capture: Evidence from Action Video Players," J Vis, 8(16), pp. 13-19, 2008.

[13] R. Li, U. Polat, W. Makous, and D. Bavelier, "Enhancing the Contrast Sensitivity Function Through Action Video Game Training," Nat Neurosci, 12(5), pp. 549-551, 2009.

[14] G. P. Caplovitz, and S. Kastner, "Carrot Sticks or Joysticks: Video Games Improve Vision," Nat Neurosci, 12(5), pp. 509-527, 2010.

[15] J.W. Karle, S. Watter, and J. M. Shedden, "Task Switching in Video Game Players: Benefits of selective Attention but not Resistance to Proactive Interference." Acta Psychol (Amst), 134(1), pp. 70-78, 2010.

[16] J. Mishra, M. Zinni, D. Bavelier, and S.A. Hillyard, "Neural Basis of Ssuperior Performance of Action Videogame Players in an Attentiondemanding Task," J Neurosci, 31(3), pp. 992-998, 2011.

[17] J. D. Chishoim, C. Hickey, J. Theecuwes, and A. Kingstone, "Reduced Attentional Capture in Action Video Game Players," Atten Percept Psychophys, 72(3), pp. 667-671, 2010.

[18] K. Clark, M.S. Fleck, and S.R. Mitroff, "Enhanced Change Detection Performance Reveals Improved Strategy Use in Avid Action Video Game Players, " Acta Psychol (Amst), 136(1), pp. 67-72, 2011.

[19] A. D. Castel, J. Pratt, and E. Drummond, "The Effects of Action Video Game Experience on the Time Course of Inhibition and Return and the Efficiency of Visual search," Acta Psychol (Amst), 119(2), pp. 217-230, 2005.

[20] G. L. West, N. Al-Aidroos, J. Pratt, "Action Video Game Experience Affects Oculomotor Performance," Acta Psychologica, 142, pp. 38-42, 2013.
[21] T. Kujala, K. Karma, R. Ceponiene, S. Belitz, P. Tukkila, M. Tervaniemi, and R. Näätänen, "Plastic Neural Changes and Reading Improvement Caused by Audiovisual Training in Reading-Impaired Children," Proc Nati Acad Sci USA, 98(18), pp. 10509-10514, 2001.

[22] R. Nouchi, Y. Taki, H. Takeuchi, H. Hshizume, Y. Akitsuki, Y. Shigemune, A. Sekiguchi, Y. Lotozaki, T. Tsukiura, Y. Yomogida, and R. Kawahima, "Brain Training Game Improves Executive Functions and Processing Speed in the Elderly: A Randomized Controlled Trial," PLOS One, 7(1), e29676, 2012.

[23] J. van Muijden, G. P. Band, and B. Hommel, "Online Games Training Aging Brains: Limited Transfer to Cognitive Control Functions." Front Hum Neurosci, 6:221, 2012.

[24] C. S. Green, and D. Bavelier, " Learning, Attentional Control, and Action Video Games, " Curr Biol. , 22(6), pp.R197-206, 2012.

[25] D. Bavelier, G. S. Green, A. Pouget, and P. Schrater, "Brain Plasticity Through the Life Span: Learning to Learn and Action Video Games, Annu. Rev. Neurosci, 35, PP. 391-416, 2012

[26] Neuroscience and Education: Issues and Oppoutunities, A Commentary by the Teaching and Learning Research Programme TERP(Teaching \& Learning Research programme) \& ESRC(economic \& Social Research council)

[27] A. M. Owen, A. Hampshire, J. A. Grahn, R. Stenton, S. Dajani, A. S. Burns, R. J. Howard, and C. G. Balllard, "Putting Brain Training to the Test," Nature, 465(7299), pp. 775 778, 2010.

[28] M. Melby-Lervåg, and C. Hulme, " Is Working Memory Training Effective? A Meta-Analytic Review." 
Developmental Psychology. Advance online 2012.doi:10.1037/a0028228

[29] W. R. Boot, D. P. Blakely, and D. J. Simons, "Do Action Video Games Improve Perception and Cognition?, “Frontiers in Psychology, 2 (226), 2011, doi: $\quad 10.3389$ /tpsyg.2011.00226

[30] U. Goswami, "Neuroscience and Education," British Journal of Educational Psychology, 74, pp. 1-14, 2004.
[31] U. Goswami, "Neuroscience and Education: from Research to Practice?" Nat Rev Neurosci, 7(5), pp. 406-11, 2006.

[32] J. Beddington, C. L. Cooper,J. Field, U. Goswami, F.A. Huppert, R. Jenkins, H.S. Jones, T. B. L. Kirkwood, B.J. Sahakian, and S. M. Thomas "The Mental Wealth of Nations." Nature, 455, pp. 1057-1060, 2008. 